\title{
Principios éticos que guían el desempeño de académicos de posgrado de la Universidad Nacional Autónoma de México
}

\author{
Ana Hirsch-Adler
}

\section{RESUMEN}

En este trabajo se da cuenta de los principales temas señalados por 34 profesores e investigadores de la Universidad Nacional Autónoma de México (unam), como respuesta a la pregunta: ¿Cuáles principios éticos guían su desempeño como académico del posgrado de la unam? Es parte del Estudio sobre la Excelencia del Profesorado del Posgrado de la unam, y los resultados se basan en una guía de entrevista aplicada entre 2017 y 2018. Los sujetos fueron seleccionados a partir de las respuestas a una de las cuatro preguntas abiertas que se incluyeron en el cuestionario de Fernández-Cruz y Romero (2010): "Mencione usted hasta tres profesores de posgrado que considere excelentes". Contestaron el cuestionario 399 académicos en 2016.

Palabras clave: principios éticos, académicos de posgrado, excelencia del profesorado, México. 
Princípios éticos que guiam o desempenho de acadêmicos de pós-graduação da Universidade Nacional Autônoma do México

\section{RESUMO}

Neste trabalho se da nota os principais temas apontados por 34 professores e pesquisadores da Universidade Nacional Autônoma do México (unam), como resposta à pergunta: quais princípios éticos guiam seu desempenho como acadêmico de pós-graduação da unam? É parte do Estudo sobre a Excelência do Professorado de Pós-graduação da unam, e os resultados se baseiam em uma guia de entrevista aplicada entre 2017 e 2018. Os sujeitos foram selecionados a partir das respostas a uma das quatro perguntas abertas que se incluíram na enquete de Fernández-Cruz e Romero (2010): "Mencione até três professores de pós-graduação que considere excelentes". Responderam à enquete 399 acadêmicos em 2016.

Palavras chave: princípios éticos, acadêmicos de pós-graduação, excelência do professorado, México.

\section{About the Ethical Principles That Guide the Performance of Postgraduate Academics at the Universidad Nacional Autónoma de México}

\section{ABSTRACT}

This paper addresses the main issues raised by 34 professors and researchers who teach in diverse postgraduate programs at the National Autonomous University of Mexico (unam), in response to the following question: What ethical principles guide your performance as a postgraduate academic at unam? It is part of the unam Graduate Teaching Excellence Study, and the results are based on an interview guide applied between 2017 and 2018. The subjects were selected from the answers to one of the four open-ended questions included in the questionnaire drawn up by Fernández-Cruz and Romero (2010): "Mention up to three graduate professors that you consider excellent". The questionnaire was answered by 399 academics in 2016 .

Key words: ethical principles, postgraduate academics, faculty excellence, Mexico. 


\section{Introducción}

El objetivo del trabajo es comunicar los principales temas señalados por 34 académicos de posgrado de la unam a la pregunta abierta ¿Cuáles principios éticos guían su desempeño como académico del posgrado de la unam? Los resultados se basan en una de las preguntas de la guía de entrevista que se aplicó en 2017 y 2018, ${ }^{1}$ como parte del Estudio sobre la Excelencia del Profesorado del Posgrado de la unam. ${ }^{2}$

Para indicar, desde el referente teórico, qué se entiende por principios éticos y los principales elementos éticos que se articulan con ellos, se retomaron autores que son reconocidos en el campo temático sobre ética profesional, tales como Augusto Hortal (2002) y Francisco Bermejo (2002), y sobre ética de la investigación como es el caso de Martín Aluja y Andrea Birke (2004), Tom Beauchamp y James Childress (2013), Adil Shamoo y David Resnik (2009), Linares (2008) y Munro y Macrina (2014). Se incorporaron también las propuestas de Marilyn y Jason Morris (2016), Jean McNiff (2016) y David Maughan (2016).

Para el trabajo empírico sobre la excelencia del profesorado universitario, se empleó el cuestionario de Fernández-Cruz y Romero (2010) de la Universidad de Granada, al que se agregaron cuatro preguntas abiertas. Éstas son: 1) ¿Cuáles considera usted que son los cinco valores básicos que la universidad debería promover con sus académicos? 2) Indique usted ¿cuáles son los cinco principales rasgos de "ser un buen profesor universitario"? 3) ¿Ciómo influyen sus proyectos de investigación en sus actividades de docencia y tutoría?, y 4) Mencione usted hasta tres profesores de posgrado que considere excelentes. El número de sujetos que contestó el cuestionario y las preguntas abiertas, en 2016, en una plataforma de Google Forms, fue de 399.
La cuarta pregunta dio como resultado una lista de 710 nombres que fueron señalados como profesores de excelencia. Los más nombrados fueron 15 académicos. De ellos, respondieron la entrevista 11 en 2017. Después, de la entrevista 12 y 13 aplicada en 2017 y las 21 realizadas en 2018, la elección recayó en la posibilidad de representar en mayor medida las cuatro áreas de conocimiento en que la unam clasifica sus 41 posgrados. Se aplicaron las guías a un total de 34 del listado de 710 .

Como sólo se contaba con los nombres, se realizó una búsqueda electrónica en bases de datos de la propia unam y del Consejo Nacional de Ciencia y Tecnología (conacyt). Con esa información se localizó a profesores e investigadores de posgrado para solicitar su participación. En todos los casos se conservó el anonimato.

\section{Aspectos teóricos Principios, virtudes y normas}

Marilyn y Jason Morris (2016) afirman que la investigación tiene tres elementos centrales: aspectos normativos, que establecen estándares mínimos para la conducta; principios éticos, que ayudan a identificar y definir asuntos éticos relevantes, y virtudes éticas, que orientan la prioridad de los asuntos relevantes. Los tratamos a continuación.

\section{Principios}

De acuerdo con Hortal (2002: 92-93):

los principios éticos son aquellos imperativos de tipo general, que nos orientan acerca de qué hay de bueno y realizable en unas acciones y de malo y evitable en otras $[\ldots]$ los principios morales no prescriben actuaciones concretas de forma directa e inmediata,

\footnotetext{
${ }^{1}$ Las primeras 13 entrevistas fueron aplicadas en 2017, por el doctor José Francisco Alanís Jiménez, que fungió como becario de posdoctorado en el proyecto de investigación, de agosto de 2015 a 2017, por parte del Consejo Nacional de Ciencia y Tecnología (México). Las otras entrevistas (21) se aplicaron en el 2018 por la doctora Karin Yovana Quijada Lovatón, que participó como becaria del Programa de Apoyo a Proyectos de Investigación e Innovación Tecnológica de la unam.

${ }^{2}$ El proyecto está financiado por el Programa de Apoyo a Proyectos de Investigación e Innovación Tecnológica de la unam, del 2017 al 2019 .
} 
más bien apuntan a los temas y metas que no hay que perder de vista a la hora de actuar y sobre todo a la hora de formular cualesquiera normas o prescripciones morales.

Beauchamp y Childress (2013: 13) indican que "los principios éticos funcionan como un marco de referencia analítico de las normas generales, que se derivan de la moralidad común [...]. Dichos principios son guías generales para la formulación de reglas específicas”. Los cuatro grandes principios que proponen son Respeto por la Autonomía, Beneficencia, No Maleficencia y Justicia.

Con respecto al primero, retomamos lo expresado por Francisco Bermejo (2002: 105) que indica que: "La palabra autonomía procede del griego: autos (sí mismo) y nomos (ley) y hace referencia a la capacidad que tiene cada cual de darse a sí mismo sus propias normas, procurando construir la propia vida a partir de ellas".

Para Beauchamp y Childress (2013:101) implica que "la propia actuación esté libre de la interferencia de otros y de las limitaciones que obstaculizan una elección significativa, tal como un inadecuado entendimiento". Para ellos, "Respetar la autonomía consiste en reconocer que los agentes tienen derecho a tener sus propios puntos de vista, a tomar decisiones y a llevar a cabo acciones que se basan en sus valores y creencias" (ibid: 106-107). Consideran también la necesidad de apoyar a otros a que puedan desarrollar su propia autonomía.

El principio de Beneficencia se refiere a la obligación moral de actuar en beneficio de otros (Beauchamp y Childress, 2013: 203). Para Bermejo (2002: 75): "La palabra beneficencia está compuesta de dos vocablos de origen latino, bene y facere, que se traducen como hacer el bien. Hace referencia a la consecución de determinados bienes específicos de la práctica profesional correspondiente". Se refiere a todas las formas de acción profesional que buscan beneficiar a otras personas.
El principio de No Maleficencia enfatiza la obligación de no infligir daño a otros y consiste en actuar de manera que no se ponga en riesgo o se lastime a las personas.

Con respecto al Principio de Justicia, Hortal (2002) afirma que la ética profesional queda incompleta si no se enmarca en la perspectiva de una ética social, que permita entender en qué contribuye o puede contribuir el trabajo de cada profesión a mejorar la sociedad. Beauchamp y Childress (2013: 250) lo interpretan "como el tratamiento justo, equitativo y apropiado" con que debe tratarse a las personas.

Bermejo (2002), Hortal (2002) y Beauchamp y Childress (2013) agregan a este principio general la necesidad de considerar específicamente la justicia distributiva. Beauchamp y Childress (2013: 250) manifiestan que se refiere "a la distribución justa, equitativa y apropiada de los beneficios y de las cargas" que se destinan para cumplir con las demandas que presentan las personas y los grupos en una sociedad determinada. "Incluye las políticas que asignan beneficios y cargas tales como la propiedad, los recursos, los impuestos, los privilegios y las oportunidades".

Para Bermejo (2002) se hacen presentes tres protagonistas: los usuarios que reclaman determinados bienes y servicios, el profesional que requiere de medios para ofrecerlos y los responsables públicos que representan al conjunto de la sociedad y que buscan conseguir un cierto equilibrio entre necesidades, exigencias y expectativas de todos.

Con un mayor énfasis en los valores, Adil Shamoo y David Resnik (2009: 5-6) consideran que: "los conceptos éticos y los principios, tales como honestidad, integridad, confianza, rendición de cuentas, respeto, confidencialidad e imparcialidad, juegan un papel central en modelar la conducta ética en la ciencia". Aseguran que los principios éticos son útiles para pensar en preguntas, problemas y decisiones de carácter ético y los definen de manera sencilla como reglas altamente generales. 
Es importante señalar que Beauchamp y Childress (2013) derivan de los principios éticos una serie de reglas. Por otro lado, Shamoo y Resnik (2009: 26) afirman que hay que distinguir entre los principios generales y los que dan cuenta de un área particular de conducta, como es el caso de la investigación científica.

Los principios éticos generales se aplican a todas las personas de una sociedad. ${ }^{3}$ Las reglas éticas especiales son sólo para las personas que ocupan roles sociales específicos, tales como determinadas posiciones, ocupaciones o profesiones. Así, cuando las personas forman parte de una profesión se comprometen explícita o implícitamente a regirse por las reglas éticas de dicha profesión.

Shamoo y Resnik (2009: 26) afirman que las teorías éticas generales y los principios sistematizan nuestros juicios sobre lo que es correcto e incorrecto y lo que es bueno y malo. Los principios éticos especiales sistematizan nuestros juicios sobre lo que es correcto e incorrecto y lo que es bueno y malo, pero con respecto a roles sociales particulares. No son simplemente una aplicación de los principios generales, sino que consideran lo que es diferente y único de los roles sociales.

Uno de los ejemplos que ponen estos autores (ibid., 27) es el siguiente: al hacer juicios acerca de la honestidad en la ciencia, consideramos el principio de ética general "ser honesto" en el contexto de la conducta científica, como es el caso de: grabar, reportar, analizar e interpretar los datos. El principio de la honestidad en la ciencia, por lo tanto, incluye elementos que son únicos en dicha actividad. Como resultado, la honestidad en la ciencia puede ser diferente de la honestidad en un diferente rol social.

\section{Virtudes éticas}

De acuerdo con Morris y Morris (2016: 202-203), las virtudes éticas se articulan, por un lado con los principios y por el otro con las normas; dan cuenta de un punto de vista positivo de la naturaleza humana y se enfocan en el desarrollo del carácter de los individuos que tienen que enfrentar dilemas morales. La ética de las virtudes enfatiza que los sujetos pueden formarse en ellas para desarrollar buenos hábitos y conductas. Afirman que los principios éticos y las virtudes éticas se complementan.

Beauchamp y Childress (2013: 378-380) consideran que "una virtud es un rasgo de carácter, moralmente bueno y elogiado, que está profundamente arraigado y que convierte a las personas en moralmente confiables $[\ldots]$ permiten a las personas discernir que deben hacer y estar motivadas a llevarlas a cabo en circunstancias particulares, sin necesidad de reglas preexistentes".

En especial, "en la vida profesional los rasgos que garantizan estímulo y admiración con frecuencia derivan de la responsabilidad de los roles [...]. La práctica profesional tiene una tradición que requiere de los profesionales el cultivo de ciertas virtudes". Priorizan las siguientes: cuidado, compasión, discernimiento, integridad, confiabilidad, veracidad y conciencia (ibid:: 33).

\section{Virtudes y valores}

Según Jean McNiff (2016: 6), los valores otorgan significado a lo que hacen los investigadores "cuando aceptamos valores particulares, como parte de las prácticas cotidianas, los internalizamos como virtudes". Manifiestan que ciertos valores son aceptados socialmente, tales como: trabajo arduo, tenacidad,

\footnotetext{
${ }^{3}$ Según Shamoo y Resnik (2009: 25) los principios generales (que coinciden con los propuestos por Beauchamp y Childress (2013) son: Autonomía: permitir que los individuos racionales tomen decisiones que conciernen a sus propios asuntos y que actúen con respecto a ellos; No Maleficencia: no dañar a otros ni a sí mismo; Beneficencia: promover su propio bienestar y el de los otros, y Justicia: tratar a las personas justamente.
} 
compasión, colegiabilidad, diálogo y consideración por los demás.

Dearing (1997, en Maughan, 2016: 21) designa que los valores compartidos en la educación superior son:

Compromiso con la búsqueda de la verdad, responsabilidad de compartir el conocimiento, libertad de pensamiento y expresión, análisis riguroso de las evidencias y el empleo de argumentos razonados para alcanzar una conclusión, voluntad de escuchar puntos de vista alternativos y juzgarlos a partir de sus méritos y compromiso en considerar las implicaciones éticas de los resultados y prácticas.

\section{Normas y reglas éticas}

Según Morris y Morris (2016: 202-203), las normas son reglas, deberes y responsabilidades y son necesarias para asegurar que la investigación tenga un estándar mínimo aceptable sobre la conducta de la investigación y se aporten los medios para hacer cumplir dichos estándares.

Beauchamp y Childress (2013: 14) indican que los principios son más generales y más comprehensivos que las reglas. Éstas últimas son más específicas en contenido y más restringidas. Plantean los siguientes tipos de reglas: a) sustantivas, tales como veracidad, confidencialidad, privacidad y fidelidad; b) de autoridad, que tratan acerca de quién debe tomar las decisiones y realizar las acciones, y c) procedimentales, que establecen los mecanismos que deben seguirse.

\section{Conductas no éticas}

Se incluyó este tema, pues varios de los entrevistados indicaron su preocupación por conductas no éticas que llevan a cabo algunos académicos y estudiantes en la unam.

Martín Aluja y Andrea Birke (2004: 100-101), con respecto a la ética de la investigación, centran su atención en las conductas no éticas y señalan, con base en una revisión de documentos internacionales, que hay un acuerdo en las tres siguientes: a) fabricación de datos, que se define como la invención de datos y experimentos que no se efectuaron; b) falsificación de datos, que se refiere a la alteración de los datos experimentales con el fin de producir un resultado que se ajuste a las expectativas de los investigadores, y c) plagio, que se establece como la apropiación de ideas, métodos innovadores, datos o cuerpo de un texto como propio, sin citar la fuente o reconocer al creador de la idea.

Como puede verse en los elementos teóricos presentados, los principios éticos se vinculan fuertemente con virtudes, reglas y valores. Esta situación se encuentra no sólo en el marco de referencia de la ética de la investigación, sino que se presenta en las respuestas de los entrevistados a la pregunta abierta ya mencionada sobre ¿Cuáles principios éticos guían su desempeño como académico del posgrado de la unam?

\section{Características generales de los entrevistados}

\section{Posgrados en donde dan clases y tutorías}

Todos los académicos entrevistados cuentan con doctorado y varios imparten clases y tutorías en más de un posgrado. Lo hacen, además, en diversas áreas de conocimiento. La unam clasifica sus 41 posgrados en cuatro grandes áreas:

- Área I: Ciencias Físico-Matemáticas y de las Ingenierías contestaron de: Ciencias Físicas, Ciencias Nucleares, Ciencia e Ingeniería de Materiales, Ciencias de la Tierra e Ingeniería.

- Área II: Ciencias Biológicas, Químicas y de la Salud, fue la que incluyó mayor número de entrevistados. Ofrecen docencia y tutorías en: Ciencias Bioquímicas, Ciencias Biomédicas, Ciencias Biológicas, Ciencias Químicas, Ciencias Médicas-Odontológicas y de la Salud, Ciencias de la Sostenibilidad, Ciencias del Mar y Limnología y Psicología.

- Área III: Ciencias Sociales, se incorporaron 
sujetos de los siguientes posgrados: Antropología, Ciencias Políticas y Sociales, Derecho, Estudios Latinoamericanos y Trabajo Social.

- Área IV: Humanidades y de las Artes, hubo representación de Arquitectura, Estudios Mesoamericanos, Historia, Filosofía, Filosofía de la Ciencia, Lingüística y Pedagogía.

\section{Edad}

En la muestra de los 34 entrevistados hay cuatro mayores de 80 años, cuatro mayores de 70,15 mayores de 60 , ocho mayores de 50 y dos de más de 40 . Una persona no indicó su edad.

\section{Género}

Mujeres, 13 y Hombres, 21.

\section{Antigüedad en la UNAM}

Coincidentemente con la edad, han trabajado durante mucho tiempo en la institución. El de más antigüedad es un emérito de 60 años de labores y después están cinco con más de 50, seis con más de 40, 12 con más de 30 , siete con más de 20 y tres con menos de 20 años de antigüedad.

\section{Pertenencia y nivel en el Sistema Nacional de Investigadores (SNI)}

Contestaron la entrevista tres profesores eméritos de este sistema, 18 del nivel más alto (III), cinco del Nivel II y 4 del Nivel I. Cuatro especificaron no ser miembros del sni en el momento de la entrevista.

\section{Pertenencia al Programa de Primas al Desempeño del Personal Académico de Tiempo Completo (PRIDE) de la UNAM}

La gran mayoría (31) cuentan con el nivel D que es el más alto del pride, dos más tienen el nivel $\mathrm{G}$ y uno no tenía este estímulo en el momento de la aplicación del instrumento. Como puede apreciarse, se trata de académicos con una enorme trayectoria en la unam.

\section{El trabajo empírico: principales asuntos basados en las respuestas de los entrevistados}

Los principales temas que se obtuvieron con base en las respuestas de los entrevistados son: Los principios y las reglas son fundamentales, Importancia de los principios generales (beneficencia y justicia), Virtudes, Reglas éticas, Valores, Fuerte identidad con la unam, Evitar conductas no éticas, La atención a los estudiantes es prioritaria, Responsabilidad de los estudiantes con la institución y la sociedad, Convivencia con los demás, El desarrollo tecnológico y científico no ha estado acompañado del desarrollo ético y Condiciones necesarias y problemas.

\section{Los principios y las reglas son fundamentales}

En opinión de los participantes, los principios éticos son esenciales y forman parte de las reglas generales de la ciencia, pues todos los investigadores los conocen y la ciencia no puede llevarse a cabo sin ellos.

Con los ejemplos que se presentan a continuación puede verse que esta idea la comparten profesores e investigadores de posgrado de las cuatro áreas en que la unam clasifica sus 41 posgrados.

la ética parte de los [...] lineamientos que da la propia universidad, es decir, las leyes universitarias, la legislación universitaria, el Estatuto y todos los reglamentos $[. .$.$] tienen que ver con derechos y obligaciones,$ [...] con el cumplimiento de los máximos objetivos que establece una institución universitaria como es la unam (entrevista 17, 2018, posgrado: Historia).

Las reglas generales en ciencia son la honestidad intelectual, la capacidad crítica y el respeto a los estudiantes y a los colegas (entrevista 4, 2017, posgrados: C. Biológicas y C. Biomédicas).

Hay ciertas reglas, estándares internacionales aceptados por nuestras comunidades y tenemos que asegurarnos de que estamos cumpliendo con la parte académica y también que estamos respetando el 
trabajo de otros miembros de la comunidad; que les estamos dando el crédito que merecen (entrevista 6, 2017, posgrado: C. Físicas).

Yo por mi lado soy de la idea de que los principios éticos son rectores de la vida de una persona. [...] Un principio que guía a los profesores [...] es que tratan de ayudar al joven a mantener una línea ética (entrevista 9, 2017, posgrados: Física, Filosofía e Ingeniería).

Como profesor siempre he tratado de tener valores que son muy ligados al trabajo científico, como el apego a la verdad, el apego al método científico, al método comprensivo de las Ciencias Sociales (entrevista 14, 2018, posgrado: C. Políticas y Sociales).

Mis principios éticos son la solidaridad hacia mi estudiantado, la no discriminación, la igualdad, no nada más la equidad, sino la igualdad de género, la no violencia, la horizontalidad y la construcción colectiva del conocimiento (entrevista 13, 2018, posgrados: C. Políticas y Sociales y Psicología).

\section{Importancia de los principios generales (Beneficencia y Justicia)}

Se indicaron ideas que pueden ligarse con el principio de Beneficencia. Estas son, por ejemplo:

El principio de Beneficencia, desde la perspectiva de que nosotros como académicos debemos buscar ese beneficio para la sociedad y también el principio de Autonomía, desde la perspectiva de ver a los estudiantes como personas dignas (entrevista 16, 2018, posgrado: Derecho).

En cualquier punto que esté uno en el mundo, es uno un actor, pero un actor crítico y debe perseguir los fines que a su juicio son los que deben perseguirse en cada momento (entrevista 1, 2017, posgrados: Antropología y Estudios Mesoamericanos).

Que estés transmitiendo un conocimiento y que sea bueno, que contribuya al bienestar de la sociedad (entrevista 3, 2018, posgrados: Ciencias de la Tierra e Ingeniería Ambiental).
En la universidad tenemos que ser líderes y pensadores, no conformarnos transmitiendo conocimientos y nada más, sino cuestionar e ir mejorando las cosas [...]. La esencia de la Ingeniería es servir a la sociedad (entrevista 2, 2018, Posgrado: Ingeniería).

Sobre el principio de Justicia, un entrevistado indicó:

Ésta es la universidad del país, a veces se pierde esto de vista. Yo en lo particular creo que esta universidad debe ofrecer la oportunidad a cualquier mexicano y a cualquier latinoamericano a que venga a estudiar. El otro elemento es la justicia, o sea la apertura y la oportunidad a todos los que quieran y que demuestren un mínimo y la justicia de no obstaculizarlo, sino al contrario, habría que identificar cuáles son sus problemas, darles la oportunidad a que lo resuelvan y ya es cuestión de la persona que esto se logre (entrevista 11, 2018, posgrados: C. Médicas, Odontológicas y de la Salud y C. Biomédicas).

\section{Virtudes}

La principal virtud que apareció en las respuestas es ser justo. Del posgrado de Derecho se plantearon para la administración pública principios de integridad, tolerancia, tenacidad y ecuanimidad (entrevista 16, 2018). Jean McNiff (2016: 6) clasifica este tipo de elementos no como principios sino como virtudes.

También se mencionó una ética de trabajo:

Que les permita entender porque una investigación avanza, porque no avanza, como resolver los problemas y no hacer los resultados a conveniencia, que realmente son plagios [...] que echan a perder un trabajo que de otra manera debió ser muy importante (entrevista 11, 2017, posgrado: C. Bioquímicas).

Aunque se señaló limitadamente, también hubo referencias con respecto a la integridad, que según Beauchamp y Childress (2013: 40) "significa solvencia, 
confiabilidad, plenitud e integración del carácter moral". Representa dos aspectos del carácter de la persona: la integración coherente de aspectos emocionales, aspiraciones y de conocimiento y la fidelidad a los valores morales y a su defensa en caso necesario.

La conciencia también se mencionó escasamente. Beauchamp y Childress (2013:42) manifiestan que un individuo actúa conscientemente si está motivado a hacer lo que es correcto, ha reflexionado sobre ello y se esfuerza por llevar a cabo este tipo de acciones.

\section{Reglas éticas}

Se presentó varias veces la de veracidad (ligada con la búsqueda de la verdad), que como ya se especificó en la parte teórica, Beauchamp y Childress (2013) la consideran una regla sustantiva.

The Norwegian National Research Ethics Committees (2016: 10) afirma que la obligación fundamental de la ciencia es la búsqueda de la verdad y que las normas tienen valor en sí mismas como guías y como principios reguladores para el propósito colectivo de trabajar para la verdad por parte de la comunidad de investigación. Uno de los entrevistados, por ejemplo, la describe como:

El ser veraz y el evitar simplificaciones conceptuales, que luego desvían el conocimiento. El desarrollar una guía crítica sobre el conocimiento [...] Entonces a esta veracidad me refiero, invitar al colega o al alumno a que piense, que no se crea que lo que está en el libro es cierto (entrevista 11, 2018, posgrados: C. Médicas, Odontológicas y de la Salud y C. Biomédicas y C. Bioquímicas).

De acuerdo con Beauchamp y Childress (2013: 303), "la veracidad en el cuidado de la salud se refiere a una transmisión de información precisa, a tiempo, objetiva y comprehensiva". Se conecta con el principio de Respeto por la Autonomía y entre los argumentos que la sostienen está la enorme importancia que tiene para el desarrollo y mantenimiento de la confianza. Dearing (en Maughan, 2016), como el primero de los valores que se comparten en la educación superior, incluye el compromiso con la búsqueda de la verdad.

\section{Valores \\ Valores en general}

Los valores que se necesitan para convivir con los semejantes. Básicamente pensarse como miembro de los distintos conglomerados con los que va coexistiendo a lo largo de la vida. [...] Entonces tratar de llevar una vida con conciencia de participación. Entenderse como uno es en realidad. [...] Lo que debemos hacer es reconocer una ética social a la que uno pertenece por ser parte del grupo [...]. En cualquier punto que esté uno del mundo, es uno un actor, pero un actor crítico (entrevista 1, 2017, posgrados: Antropología y Estudios Mesoamericanos).

\section{Valores múltiples}

Me parece que son básicamente integridad, honestidad académica, respeto, tolerancia a los alumnos y responsabilidad social y ambiental en el salón de clase (entrevista 10, 2017, posgrados: C. Químicas, C. Bioquímicas e Ingeniería de Materiales).

Los valores fundamentales son responsabilidad, intentar ser justo, buscar la verdad, la honradez que implica que lo que digas sea apegado a la realidad (entrevista 3, 2018, posgrados: Ciencias de la Tierra e Ingeniería Ambiental).

\section{Valores más señalados}

\section{Honestidad}

Acerca de los valores, el más mencionado es el de honestidad intelectual y científica. Se expresaron ideas como las siguientes:

La honestidad intelectual se entiende como presentar las cosas como las ve, las entiende, las obtiene, lo 
más claramente posible. La honestidad es preguntarse lo que obtiene y cómo lo interpreta (entrevista 2, 2017, posgrados: C. Bioquímicas y C. Biomédicas).

Tiene que ver con reconocer los límites que se tienen con respecto al conocimiento (entrevista 5, 2017, posgrado: C. Físicas).

Yo en lo particular creo que la honestidad de un investigador es siempre fundamental, como investigador, como profesor y como individuo (entrevista 19, 2018, posgrados: Filosofía, Filosofía de las Ciencias e Ingeniería y C. de la Computación).

La honestidad académica es muy importante. Yo lo podría resumir en una frase; no hacernos tontos, no hacer en que hago y no hago, no fingir que publico y no publico, no fingir que sé y no sé, todo lo que es la simulación (entrevista 17, 2018, posgrado: Historia).

\section{Respeto}

También se mencionó varias veces el valor de respeto, con respecto a otros miembros de la comunidad, a los estudiantes y al público de referencia del trabajo académico. Algunos ejemplos son:

Yo entiendo el respeto no como algo jerárquico, sino equitativo, yo voy a tratar a las personas con las que interactuó de la misma manera que yo me sentiría a gusto que me trataran a mi (entrevista 7, 2018, posgrado: Psicología).

Uno es un profundo respeto. Yo creo que hay que respetar al público que uno tiene. Creo que el respeto es fundamental, no importa quién sea tu interlocutor, quién sea tu grupo, quien sea tu auditorio (entrevista 5, 2017, posgrado: C. Físicas).

Respeto, los alumnos son personas y los tengo que respetar plenamente. El trato debe ser cordial, justo y parejo (entrevista 5, 2018, posgrado: C. de la Tierra).

\section{Responsabilidad y compromiso}

Se indicaron ambos términos en general y también en referencia a cumplir siempre con las obligaciones y al compromiso social y del conocimiento.
El cumplir siempre con mi obligación, el nunca faltar, darles las reglas desde el principio a los estudiantes y decirles la clase va a ser de esta manera (entrevista 8, 2018, posgrado: C. Bioquímicas).

La parte ética de asignar un trabajo a los estudiantes, buscar nuevas formas de evaluar, discutiéndolas con los estudiantes, planteando alternativas (entrevista 2, 2018, posgrado: Ingeniería).

La parte ética es estar bien preparados, cosas tan triviales como ser puntuales [...] y actualizar nuestras clases [...] yo creo que la parte ética implica una autocrítica y un autoanálisis, porque es una responsabilidad dar clases (entrevista 9, 2018, posgrado: de C. Biomédicas).

Compromiso y acompañamiento con los estudiantes (entrevista 20, 2018, posgrado: Pedagogía).

$\mathrm{Si}$ te comprometes, por ejemplo a ser sinodal o asesora de una tesis, pues, participar activamente (entrevista 12, 2018, posgrados: Estudios Latinoamericanos y Trabajo Social).

The Norwegian National Research Ethics Committees (2016), en referencia a la investigación, considera que tiene una responsabilidad social, ya sea de tipo instrumental para las decisiones sociales, crítica como fuente de diversas elecciones de cursos de acción, o deliberativas como un insumo del conocimiento.

\section{Fuerte identidad con la UNAM}

Resultó notoria la fuerte identidad institucional que mostraron los investigadores con respecto a la unam. Se indicaron comentarios como los siguientes:

Creer en el proyecto de la unam (entrevista 5, 2017, posgrado: C. Físicas).

En realidad es la identidad institucional, conocer su visión y misión y el objetivo con que se fundó la Universidad Nacional (entrevista 6, 2018, posgrado: Psicología).

Somos parte de una sociedad [...] tenemos que dar el ejemplo, especialmente si estamos en esta 
universidad que es la Universidad Nacional (entrevista 6, 2017, posgrado: C. Físicas).

La universidad tiene la obligación de luchar por los valores éticos más importantes, más fundamentales, y lo hace en la medida de sus posibilidades. Los profesores deberían también hacer eso (entrevista 9, 2017, posgrados: Física, Filosofía e Ingeniería).

\section{Evitar conductas no éticas}

El plagio fue la conducta no ética más señalada.

En cuanto a la docencia en particular pienso que hay cosas inaceptables como el plagio. ¿Qué noto en las nuevas generaciones, por ejemplo?, que algunos estudiantes desaparecen sin avisar, cosa que no pasaba en el pasado (entrevista 3, 2017, posgrados: C. Biológicas, C. Biomédicas y C. Bioquímicas).

Lamentablemente lo veo en mis estudiantes y mis colegas, que repiten cinco veces el contenido de un mismo artículo y lo publican por aquí y por allá. No se dan cuenta que destruyen su propio trabajo al hacer eso [...] Considero que toda investigación que no es original es deshonestidad con la universidad, lo considero de poca ética profesional. Hay que preparar una buena clase y no venir a fingir con los estudiantes (entrevista 17, 2018, posgrado: Historia).

Bueno, desde luego, el evitar pretender algo que no hizo uno, apropiárselo que sería de lo más grave (entrevista 2, 2018, posgrado: Ingeniería).

\section{La atención a los estudiantes es prioritaria}

Es clara la idea de la responsabilidad que tenemos los profesores e investigadores de la unam con los estudiantes. Algunos de los ejemplos son los siguientes:

Implica ese sentido de respeto hacia las personas, hacia los estudiantes, el reconocer que cada estudiante tiene diferentes posibilidades $[\ldots]$ y darle su lugar $[. .$.$] nunca subestimarlos, eso es un principio$ para mi (entrevista 20, 2018, posgrado: Pedagogía).
La atención a los estudiantes es prioritaria y de ahí deriva nuestro trabajo de investigación. Nuestro eje es generar recursos humanos altamente especializados (entrevista 9, 2018, posgrado: C. Biomédicas).

Considerar también el trabajo que hacen las alumnas y los alumnos, darles el crédito en lo que hacen (entrevista 12, 2018, posgrados: Estudios Latinoamericanos y Trabajo Social).

Desde el punto de vista de la labor de la investigación y desde el punto de vista de la formación de los estudiantes, pues ha sido darles su lugar, darles todo el reconocimiento y el crédito por su trabajo (entrevista 4, 2018, posgrados: C. Físicas y C. Químicas).

Lo que yo quiero remarcar, más que principios éticos $[\ldots]$ fue el ejemplo que me han dado mis profesores, respetaban mucho a los estudiantes y trataban de pulir y sacar lo mejor de ti (entrevista 19, 2018, posgrados: Filosofía, Filosofía de la Ciencia e Ingeniería y C. de la Computación).

\section{Responsabilidad de los estudiantes con la institución y la sociedad}

Hubo dos académicos que señalaron la importancia de que los estudiantes se responsabilicen con la institución y con la sociedad. Dijeron lo siguiente:

Es inaceptable que la universidad les dé todo y que ni siquiera sepan cómo es que están aquí de esta manera. La parte de la responsabilidad del estudiante hacia su institución y hacia su sociedad que lo está financiando es muy importante (entrevista 10, 2017, posgrados: C. Químicas, C. Bioquímicas e Ingeniería de Materiales).

Ellos tendrían compromisos como alumnos, y hablando del posgrado, tendrían que reconocer la idea de que tienen una beca, en una universidad pública que recibe impuestos de nosotros (entrevista 13, 2017, posgrados: Filosofía de la Ciencia y C. Políticas y Sociales. 


\section{Convivencia con los demás}

Los ejemplos son los siguientes:

Compartir lo que estamos encontrando (entrevista 14, 2018, posgrados: C. Físicas y C. Químicas).

Considerar que hay un gran potencial en los otros (entrevista 2, 2018, posgrado: Psicología).

No ignorar el trabajo de los demás (entrevista 7 , 2017, posgrados: C. Bioquímicas y G. Biomédicas).

Hacer un trabajo de publicación tiene que ser el fruto del esfuerzo de uno o varios colaboradores [...]. Por eso es importantísimo, éticamente hablando, que reconozcan el trabajo de los demás. Hay que conocer y darle a cada quien el crédito que merece en las investigaciones que va uno presentando en los cursos (entrevista 7, 2017, posgrado: C. Físicas).

Como se enunció previamente, McNif (2016: 6), entre los valores aceptados socialmente, incluye la colegiabilidad, el diálogo y la consideración por los demás. Dearing (en Maugham, 2016), entre los valores compartidos en la educación superior, incorpora la responsabilidad de compartir el conocimiento y la voluntad de escuchar puntos de vista alternativos.

\section{El desarrollo tecnológico y científico no ha estado acompañado del desarrollo ético}

Uno de los entrevistados planteó, sin poner ejemplos, que hay un desarrollo tecnológico y científico enorme, pero que no ha estado necesariamente acompañado de un correspondiente desarrollo ético (entrevista 9, 2017, posgrados: Física, Filosofía e Ingeniería).

Al respecto, Hortal (2002: 61) considera al impresionante avance de la tecnología como una de las mediatizaciones que afectan a todas las profesiones. Afirma que "ser competentes técnicamente es una condición necesaria, aunque no suficiente, para ser moralmente responsables en el ejercicio profesional". Agrega que cuando se difunde la mentalidad técnica se corre el riesgo de que se inhiba la responsabilidad ética de los profesionistas.

Con respecto a este tema, Jorge Enrique Linares (2008: 365) indica que: "la tecnología ha dejado de ser un mero instrumento ${ }^{4}$ para convertirse en un entorno determinante de medios y fines. El mundo tecnológico del que depende ahora la humanidad entera se ha convertido en una mediación universal y en el horizonte de las relaciones cognoscitivas y pragmáticas entre el ser humano y la naturaleza; es, pues, un sistema-mundo que domina la vida social".

Afirma que las tecnociencias han provocado diversos problemas ético-políticos, pues han generado nuevas posibilidades en la transformación de la naturaleza, lo que ha implicado diversos grados de riesgos y de efectos imprevisibles: "la creciente autonomía de la actividad tecnológica con respecto al resto de los sistemas sociales, la cultura y los valores ético-políticos de la sociedad contemporánea $[\ldots]$ ha llevado a un paralelo declive de su conciencia y responsabilidad colectiva" (Linares, 2008: 388). ${ }^{5}$

Cindy L. Munro y Francis L. Macrina (2014: 361-383) indican que los científicos tienen responsabilidades con la comunidad científica y con la sociedad. Incluyen llevar a cabo la investigación con honestidad e integridad, cumpliendo con las leyes y las normas y con el respeto que debe darse a los sujetos que participan en las investigaciones. También ser precisos y responsables al reportar los resultados y dar cuenta de los conflictos de interés y de los recursos. Ponen como ejemplos de riesgos en los avances, a los experimentos relacionados con la tecnología del adn como parte de la ingeniería genética y al Proyecto del Genoma Humano.

\footnotetext{
${ }^{4}$ Las cursivas son del autor.

${ }^{5} \mathrm{El}$ autor pone como ejemplos el Proyecto Genoma Humano, la investigación sobre inteligencia artificial, la creación de redes cibernéticas, la Internet, la robótica, las tecnologías de realidad virtual y la ingeniería genética (Linares, 2008: 378-379).
} 


\section{Condiciones necesarias y problemas}

Como condiciones necesarias para desarrollar un buen trabajo se registraron las siguientes: espacio, tiempo, financiamiento y recursos, y publicar trabajos originales e inéditos.

Algunos de los problemas marcados son: falta de tiempo para modernizar las clases, presión por publicar y llenar diversos informes para las dependencias universitarias y nacionales, las conductas no éticas y que la moral social se ha deteriorado. Varios académicos indicaron que notan una falta de responsabilidad en la docencia. Uno de los ejemplos es el siguiente:

A veces la unam puede ser muy laxa [...] profesor que viene o no viene, no avisa o manda a su ayudante o llega media hora tarde. Uno de los mayores problemas del país es que las normas no se cumplen, los compromisos no se cumplen (entrevista 13, 2017, posgrados: Filosofía de la Ciencia y C. Políticas y Sociales).

Se manifestaron cuestiones negativas, tales como la falta de interés de la institución en la docencia (entrevista 5, 2017, posgrado: C. Físicas), el rezago de los reglamentos y la necesidad de reorganizar las estructuras académicas. Se dijo que la unam se mueve lentamente y que hay procesos que son muy dificiles de cambiar por las consecuencias políticas internas que se producen cuando se busca hacer transformaciones relevantes (entrevista 10, 2017, posgrados: C. Químicas, C. Bioquímicas e Ingeniería de Materiales).

\section{A manera de cierre de la parte empírica}

Como ya se había mencionado en el rubro acerca de las características generales de los entrevistados y en los ejemplos que se expusieron en cada uno de los temas, puede notarse que la gran mayoría trabaja en varios de los posgrados ubicados por la unam en su propia área de conocimiento y también en otras.
Consideramos que ese hecho puede ser un elemento que contribuye a que - en términos de los principios éticos y de los temas que con ellos se asocian- no hayamos detectado grandes diferencias en lo expresado por los académicos de las cuatro áreas.

Pudimos darnos cuenta, sin embargo, de algunos elementos distintivos. Uno de ellos es en el área de Ciencias Biológicas, Químicas y de la Salud. Se trata específicamente de la investigación experimental y del énfasis en generar resultados reproducibles y verificables. Por ejemplo:

Cuando informas algo en una revista, tienes que poner resultados que son reproducibles y absolutamente verificables (entrevista 10, 2018, posgrado: Ciencias Químicas).

El trabajo es importante dependiendo del impacto que tenga, pero algo que es fundamental es que el trabajo sea reproducido por los medios, entonces, trata uno de presentarlo de la mejor forma posible para que otros lo hagan y lo reproduzcan (entrevista 2, 2017, posgrados: C. Bioquímicas y C. Biomédicas).

Los principios éticos son fundamentales y parten de desarrollar la experimentación con la mayor rectitud posible (entrevista 11, 2017, posgrado: C. Bioquímicas).

No es sorpresa que en las Ciencias Sociales y en las Humanidades y las Artes no se haya hecho alguna referencia con respecto a los procesos de experimentación y a la reproductibilidad de los resultados. De acuerdo con The Norwegian National Research Ethics Committees (2016), en estas disciplinas el involucramiento y la interpretación son con frecuencia partes integrales del proceso de investigación. Las diversas aproximaciones y posiciones teóricas pueden permitir interpretaciones disímiles y razonables del mismo material.

Un asunto que se presenta en algunos posgrados de estas áreas, son los estudios sobre género (en 
entrevista 12, 2017, posgrado: Estudios Latinoamericanos y entrevista 13, 2018, posgrados: Ciencias Políticas y Sociales y Psicología).

\section{A modo de conclusión}

La gran mayoría de los entrevistados, como ya se dijo, tienen una larga y exitosa trayectoria en la unam y han llevado a cabo una importante labor en la formación de nuevos investigadores. Es evidente la fuerte identidad y compromiso que tienen con la institución, aun cuando algunos indican que hay profesores que no cumplen cabalmente con sus obligaciones.

Con respecto a los profesores e investigadores elegidos para ser entrevistados, resultó de gran interés que muchos de ellos den clases y tutorías en posgrados de distintas áreas de conocimiento. Fue gratificante, también, comprobar que aspectos centrales de la ética profesional y de la ética de la investigación estuvieron presentes en sus respuestas.

Los argumentos de los teóricos considerados con respecto a los principios éticos tienen un elevado nivel de abstracción, a diferencia de lo expresado por los entrevistados, que en general tienen opiniones más cercanas a su trabajo académico. Así, los autores dan cuenta de cuatro grandes principios, que aunque no pueden aplicarse tal cual en situaciones concretas, conforman un marco de referencia para la toma de decisiones éticas. Los señalados por los sujetos se vinculan estrechamente con sus funciones de investigación, docencia, tutorías y difusión.

Como el tema de los principios éticos tiene un carácter general, encontramos contestaciones similares, en algunos asuntos, por parte de los entrevistados de posgrado de las cuatro grandes áreas de conocimiento. Hay desde luego diferencias, pero nos pareció significativo encontrar ideas que se comparten y que podrían ser relevantes para mejorar aún más la vinculación entre posgrados de áreas diferentes.

Resultó fructífero aproximarse a la comprensión de las maneras en que se articulan los principios éticos con los asuntos que les dan sustento, como son las reglas, las virtudes y los valores. Aunque se partió de los elementos teóricos para organizar los diversos temas expresados por los entrevistados, encontramos ideas que enriquecieron lo planteado previamente y asuntos que no habíamos considerado, de carácter más práctico, tales como conductas no éticas y problemas que se presentan en sus actividades y en el funcionamiento de la propia universidad. 


\section{Referencias}

Aluja, M. y A. Birke (2004), "Panorama general sobre los principios éticos aplicables a la investigación científica y la educación superior", en M. Aluja y A. Birke (coords.), El papel de la ética en la investigación científica y la educación superior, México, Fondo de Cultura Económica/Academia Mexicana de Ciencias, pp. 87143.

Beauchamp, T. y J. Childress (2013), Principles of biomedical ethics [7a. ed.], Nueva York, Oxford University Press.

Bermejo, F. (2002), La ética del trabajo social, Bilbao, España, Desclée de Brouwer.

Fernández Cruz, M. y M. Romero (2010), "Indicadores de excelencia docente en la Universidad de Granada", Revista Portuguesa de Pedagogía, vol. 44, núm. 1, pp. 83-117, <https://digitalis.uc.pt/pt-pt/ node/106201?hdl=4807> [Consulta: agosto de 2015].

Hortal, A. (2002), Ética general de las profesiones, Bilbao, España, Desclée de Brouwer.

Linares, J. E. (2008), "Hacia una ética para el mundo tecnológico [Segunda Parte]", en Ética y Mundo Tecnológico, México, unam-Facultad de Filosofía y Letras/Fondo de Cultura Económica, pp. 365-388.
Maughan, D. (2016), "Values in higher education. Articulation and action", en J. McNiff (ed.), Values and virtues in higher education research. Critical perspectives, Nueva York, Routledge, pp. 19-32.

Mcniff, Jean (2016), "Introduction", en J. McNiff (ed.), Values and virtues in higher education research. Critical perspectives, Nueva York, Routledge.

Morris, M. yJ. Morris (2016), "The importance of virtue ethics in the irb (Institutional Review Boards)", Research Ethics, vol. 12, núm 4, pp. 201-216, <journals. sagepub.com/doi/pdf/10.1177/1747016116656023> [Consulta: agosto 2018].

Munro, C. y F. Macrina (2014), "Science, technology and society [Chapter 11]", en F. Macrina, Scientific integrity. Text and cases in responsible conduct of research [4a. ed.], Washington, dc, asm Press, pp. 361-383.

Norwegian National Research Ethics Committees, The (2016), Guidelines for Research Ethics in the Social Sciences, Humanities, Law and Theology [4a. ed.], Oslo, <www. etikkom.no> [Consulta: diciembre 2018].

Shamoo, A. y D. Resnik (2009), Responsible conduct of research [2a. ed.], Nueva York, Oxford University Press.

\section{Cómo citar este artículo:}

Hirsch-Adler, Ana (2019), "Principios éticos que guían el desempeño de académicos de posgrado de la Universidad Nacional Autónoma de México", en Revista Iberoamericana de Educación Superior (RIES), México, UNAM-IISUE/Universia, vol. x, Núm. 29, pp. 143-157, DOI: https://doi.org/10.22201/iisue.20072872e.2019.29.528 [consulta: fecha de última consulta]. 